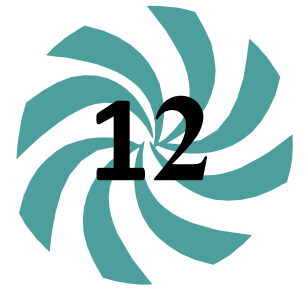

Tecnociencia, Vol. 23, $\mathrm{N}^{\circ} 1: 238-261$

Enero-Junio 2021

\title{
RIQUEZA DE ESPECIES DE AVES DE LA SIERRA LLORONA, PROVINCIA DE COLÓN, PANAMÁ
}

\author{
Nelson Guevara ${ }^{1,2}$
}

${ }^{1}$ Centro de Investigación y Capacitación en Conservación de la Biodiversidad, Sociedad Mastozoológica de Panamá (SOMASPA). ${ }^{2}$ Grupo Biológico Biomundi. email: bio.mundi18@gmail.com;

\section{RESUMEN}

Se presenta un listado sobre la riqueza de aves de La Sierra Llorona, provincia de Colón, realizado entre octubre del 2019 y febrero del 2020. El estudio se realizó en áreas de libre acceso como carreteras y vías públicas, y zonas boscosas de terrenos privados. Para este estudio se utilizó el método de búsqueda generalizada, con un total de 20 muestreos, con esfuerzo de 240 horas totales, distribuidas en 180 horas de día y 60 horas de noche. Se registraron un total de 119 especies, 37 familias y 18 órdenes, siendo Passeriformes con 66 especies y 15 familias el taxón más representativo. Las aves residentes con 90 especies superaron a las migratorias con 29 especies. Además, se registraron 24 especies importantes en conservación, según los listados presentados por el Ministerio de Ambiente de Panamá y la IUCN siendo Accipitriformes, Apodiformes y Galliformes los órdenes más representativos.

\section{PALABRAS CLAVES}

Aves, riqueza de especies, especies residentes, especies migratorias, Sierra Llorona

\section{WEALTH OF SPECIES OF BIRDS OF THE SIERRA LLORONA, PROVINCE OF COLÓN, PANAMA.}

\begin{abstract}
A list of the bird richness of La Sierra Llorona, province of Colón, is presented between October 2019 and February 2020. The study was conducted in open access areas such as roads and public roads, and wooded areas of private land. For this study, the generalized search method was used, with a total of 20 samples, with an effort of 240 total hours, spread over 180 hours a day and 60 hours at night. A total of 119 species, 37 families and 18 orders were recorded, with Passeriformes with 66 species and 15 families being the most representative taxon. Resident birds with 90 species outperformed migratory birds with 29 species. In addition, 24 important species were
\end{abstract}

Tecnociencia, Vol. 23, $N^{\circ} 1$ 
recorded in conservation, according to the lists submitted by the Ministry of Environment of Panama and the IUCN being Accipitriformes, Apodiformes and Galliformes the most representative orders.

\section{KEYWORDS}

Birds, species richness, resident species, migratory species, Sierra Llorona

\section{INTRODUCCIÓN}

En las últimas décadas, uno de los mayores éxitos en la protección de las áreas naturales es haber detenido la expansión de los potreros a costa de los bosques (Heckadon et al., 1999). Durante la década de 1950, se detuvo la expansión de la frontera agropecuaria la cual destruyó más del $50 \%$ de la superficie boscosa de la Cuenca, actualmente con una cobertura boscosa de 158,000 hectáreas (47\% de su superficie total) se mantiene en una gradual recuperación con el 69\% de sus bosques protegidos por las leyes panameñas desde 1980 (Heckadon et al., 1999).

Actualmente la biodiversidad del mundo ha disminuido debido a un desarrollo sostenible que degrada los hábitats naturales y conduce a las especies a la extinción, siendo las aves uno de los grupos más afectados a estos cambios (Birdlife, 2018). A su vez, las aves son importantes indicadores de la salud de los ecosistemas; los cambios en sus poblaciones reflejan cambios en los hábitats, por ejemplo, en la calidad del agua, en la presencia de enfermedades y en el clima (Del Coro et al., 2019). Las aves proporcionan servicios ambientales invaluables, como el control de plagas, la dispersión de semillas, la polinización y ayuda en la economía de varios países debido al turismo por observación de aves (Pereyra, 2019).

En la región de Santa Rita de la provincia de Colón se encuentra la Sierra Llorona, la cual colinda con el Parque Nacional Chagres (Gaceta Oficial, 2017; SOMASPA, 2019). Esta forma parte del Corredor Biológico Colón, brindando conectividad entre varias zonas boscosas del Parque Nacional Chagres, el Parque Nacional Soberanía y el lago Gatún ofreciendo hábitats a una gran variedad de animales, contribuyendo a los procesos ecológicos y conservación de varias especies en peligro debido a la fragmentación de los bosques. Además, 
esta zona brinda protección a la cuenca del Río Gatún (SOMASPA, 2019).

La Sierra Llorona abarca una superficie de $338 \mathrm{~km}^{2}$, con un área central de $138 \mathrm{~km}^{2}$ y una cobertura boscosa aproximadamente del $75 \%$ de su superficie. Sus zonas boscosas están compuestas principalmente por bosques secundarios y bosques maduros (SOMASPA, 2019). Hasta el momento, no se ha realizado una caracterización de las aves de la zona, la cual también ha sido severamente alterada por actividades antropogénicas como la deforestación y ganadería. El objetivo principal del presente estudio es el de brindar información general sobre varios aspectos de la avifauna de la Sierra Llorona como su riqueza de especies y estado de conservación de éstas, lo cual puede contribuir a la decisión responsable de medidas para la conservación y cuidado de la biodiversidad de este sitio conocido de la provincia de Colón.

\section{MATERIALES Y MÉTODOS}

\section{Área de estudio}

El estudio se realizó en la localidad de Sierra Llorona $\left(9^{\circ} 20^{\prime} 08^{\prime \prime} \mathrm{N}\right.$ 7947'02" W), distrito de Santa Rita, Provincia de Colón (Fig. 1). Cuenta con un clima tropical muy húmedo, caracterizado por una lluvia constante durante todo el año. En el mes más seco la precipitación es mayor a los $60 \mathrm{~mm}$. La temperatura media del mes más fresco oscila entro los 18 y $23^{\circ} \mathrm{C}$; la diferencia entre temperaturas con relación al mes más cálido es aproximadamente menor a $\operatorname{los} 5^{\circ} \mathrm{C}$, clasificación según Köpen (ETESA, 2007).

Según Holdridge (1979) Sierra Llorona está ubicada en la zona de vida de Bosque húmedo tropical (bh-T) caracterizada por una temperatura media anual que oscila entre los 24 y $26^{\circ} \mathrm{C}$, con una vegetación de tipo bosque perennifolio ombrófilo tropical, latifoliado de tierras bajas bastante intervenido en regeneración, clasificación según la UNESCO, 2000 (ANAM, 2010). La altura de Sierra Llorona oscila entro los 100 y 300 metros sobre el nivel del mar (s.n.m) con pendientes entre los $4^{\circ} \mathrm{y}$ $15^{\circ}$ (ANAM, 2010). 


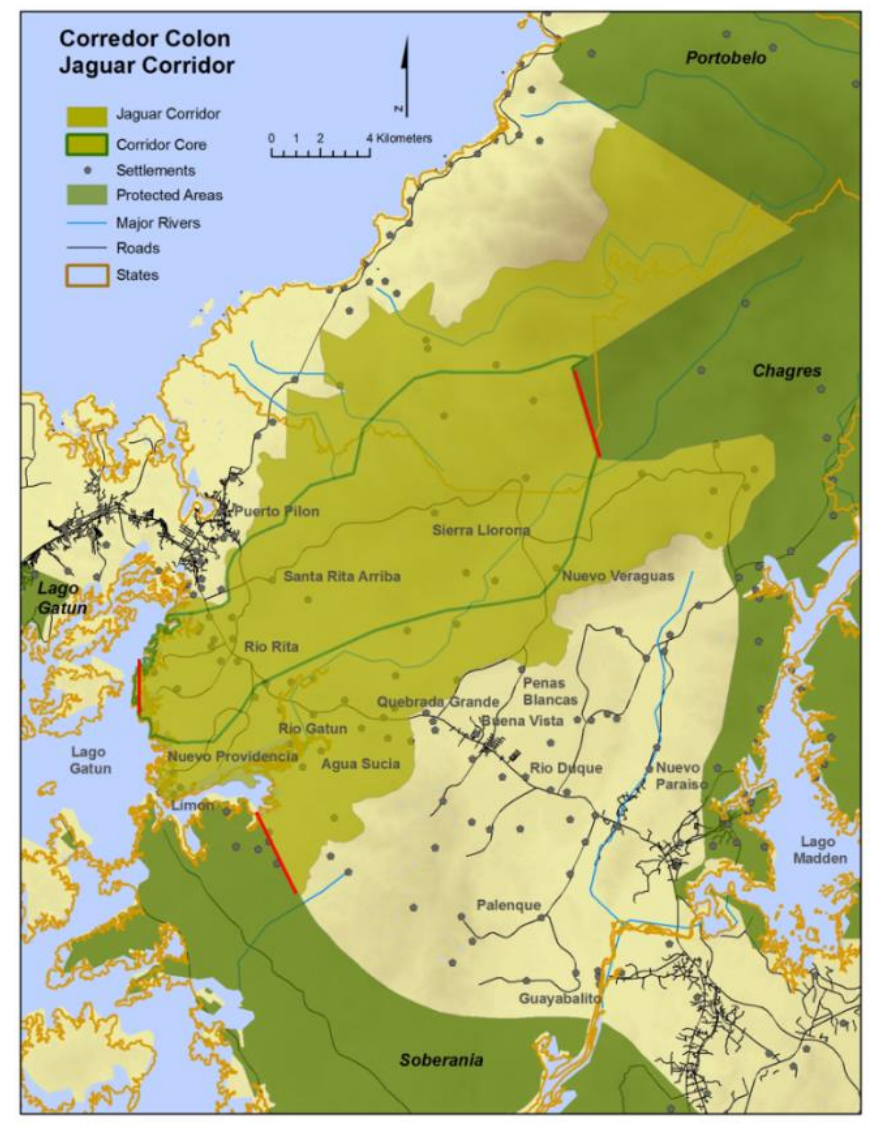

Fig. 1. Vista panorámica del área de estudio (Sierra Llorona). Parte del Corredor biológico, Corredor Colón Jaguar. Línea verdad: Área de interés del estudio, líneas rojas: límites del territorio con áreas protegidas. Mapa proporcionado por la Sociedad Mastozoológica de Panamá (SOMASPA).

\section{Registro de las especies}

El registro de las especies se realizó desde el sábado cinco de octubre del 2019 hasta el jueves 13 de febrero del 2020, mediante el método de búsqueda generalizada (Polanco et al., 2015). Las observaciones se hicieron en periodos de tres a cinco días por mes con un total de 20 días de muestreo, incluyendo el periodo de aves migratorias en Panamá. Se realizaron recorridos entre 5 a $10 \mathrm{~km}$ siguiendo las áreas de libre acceso como carreteras y vías públicas, y zonas boscosas de terrenos privados lo cual permitió realizar búsqueda en las áreas boscosas del lugar comprendidas en su mayoría por una primera zona de bosque secundario 
seguido de bosque maduro. La búsqueda se realizó de día entre las 7:00 y 16:00 horas, y durante la noche entre las 19:00 y 22:00 horas, anotando la información obtenida mediante la observación directa de las especies y en algunos casos mediante los cantos. Los registros fueron realizados por un solo observador.

Para la identificación de las especies se utilizaron binoculares Nikon MONARCH M511 8X42 6.3 ${ }^{\circ}$ waterproof, cámara profesional Canon Rebel T6 y las guías de Ridgely y Gwynne (1993) y Angehr \& Dean (2010). Para el ordenamiento filogenético de las especies se siguió el arreglo taxonómico del listado de AVIBASE propuesto por Lepage (2020), los nombres comunes de las especies fueron colocados según AUDUBON (2019). La clasificación de las aves en residentes y migratorias se basó en Angehr \& Dean (2010). Finalmente, la categoría de amenaza o conservación de las especies fue determinada por la Gaceta Oficial $\mathrm{N}^{\circ}$ 28187-A de la resolución $\mathrm{N}^{\circ} \mathrm{DM}-0657-2016$, establecida por el Ministerio de Ambiente de la República de Panamá y por el listado rojo de las especies protegidas de la Unión Internacional para la Conservación de la Naturaleza, por sus siglas en ingles IUCN.

\section{Análisis de datos}

Se utilizo el programa Microsoft Excel 2019 para el ordenamiento de los registros, tabulación de los datos y confección de tablas. Para el análisis estadístico de los datos y confección de la curva acumulativa de las especies se utilizó el programa EstimateS 9 Windows (Moreno, 2001). Se utilizaron los estimadores Chao1 y Ace para estimar el porcentaje de riqueza de las especies obtenidas en el lugar, aplicando datos de ausencia y presencia, donde el estimador Chaol es el más riguroso (Villareal et al., 2004).

\section{RESULTADOS Y DISCUSIÓN}

Esfuerzo de muestreo

El esfuerzo total de trabajo fue de 240 horas/hombres. La curva acumulativa de especies indica que se logró registrar un número representativo de aves de Sierra Llorona, según lo observado durante las horas de muestreo, ya que durante los últimos días del estudio no hubo un aumento notable en el registro de nuevas especies. Se obtuvo aproximadamente el $85 \%$ de las especies según el estimador no 
paramétrico Ace Mean y el $69 \%$ de las especies según el estimador no paramétrico Chao1, el cual indica que para la zona estudiada se puede registrar un total aproximado de 172 especies (Fig. 2). En este sentido podemos afirmar la efectividad del método de búsqueda generalizada, como mencionan Stiles y Rosselli (1998). Este método permite llevar a cabo un inventario más eficaz sobre la riqueza de especies de un área determinada. Además, un método apropiado para compilar información si se dispone de poco tiempo, logrando registrar aves que no son detectadas por otros métodos debido su forma de desplazamiento o espacio que ocupan dentro de la zona (Ralph et al., 1996).

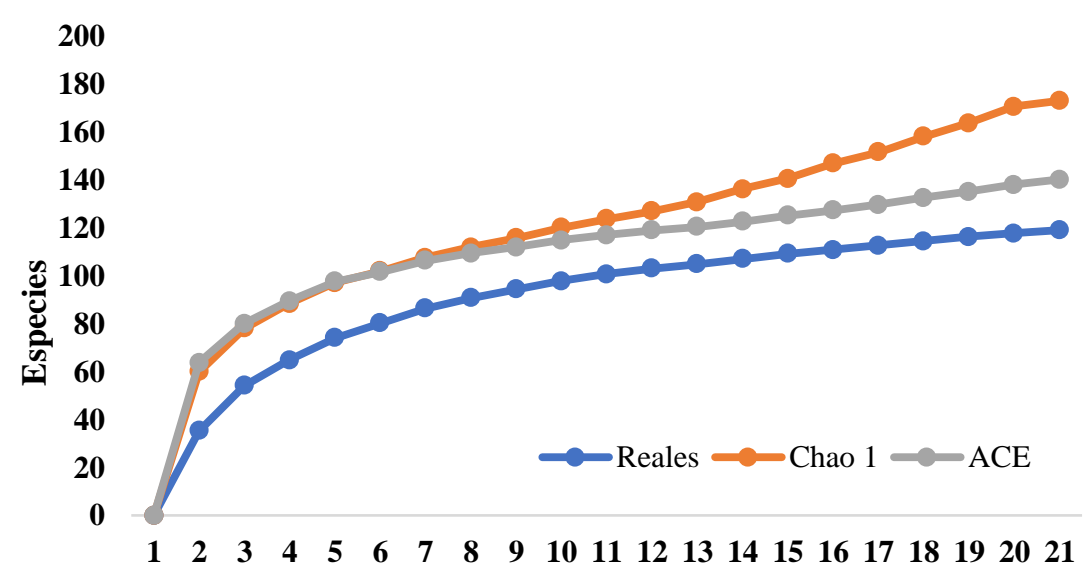

Días

Fig.2. Curva acumulativa de especies registradas en Sierra Llorona, provincia de Colón.

Riqueza de especies

En total, se registró 119 especies, 37 familias y 18 órdenes (Cuadro 1, Anexo 1). El total de especies registradas corresponde al $12 \%$ de las aves conocidas para Panamá, el cual posee un total de 1009 especies de aves (AUDUBON, 2019).

Al comparar el total de especies con áreas aledañas (Cuenca del Canal, Parque Nacional Soberanía, Parque Nacional Chagres y la Comunidad de Quebrada Ancha) se observa un número mayor de especies en la Cuenca del Canal (145 especies) (Tejera et al., 1995), el Parque 
Nacional Chagres (396) (Fundación Chagres, 2020) y el Parque Nacional Soberanía (402 especies) (Gaceta Oficial, 2017) en comparación con la riqueza de Sierra Llorona. Sin embargo, la Comunidad de Quebrada Ancha reporta un número menor de especies (25) (Cuadro 1). Esta diferencia puede deberse a que las zonas de la Cuenca del Canal y el Parque Nacional Soberanía llevan muchos más años de estudio relacionados con la avifauna de estos lugares, además de presentar una menor presión por actividades antropogénicas como la ganadería y la deforestación. Sin embargo, el número menor de especie en la Comunidad de Quebrada Ancha puede deberse a que el esfuerzo y tipo de muestreo (registro de especies por entrevistas) en esta localidad no haya sido proporcional al tamaño y complejidad de éste, debido a que las especies solo fueron registradas por entrevistas a un número de 25 personas mediante laminas ilustradas (Contreras y Yanguez, 2017). Es posible que el sesgo del conocimiento de las especies de aves por parte de los pobladores provocará el bajo registro de especies. Como mencionan Araúz y González (2010) factores como un mal manejo de métodos y tiempo de muestreo puede influir negativamente en la obtención de datos sobre las aves de una región. Además, posiblemente la riqueza de especies de La Sierra Llorona este influenciado principalmente por los Parques Nacionales Chagres y Soberanía debido a la conectividad que brinda entre estos.

Cuadro 1. Aves totales registradas en La Sierra Llorona y tres áreas aledañas.

\begin{tabular}{|l|c|c|}
\hline Área de estudio & $\begin{array}{l}\text { Total, de } \\
\text { especies }\end{array}$ & Esfuerzo de muestro \\
\hline $\begin{array}{l}\text { Sierra Llorona (estudio } \\
\text { actual) }\end{array}$ & 119 & 240 horas/hombre \\
\hline $\begin{array}{l}\text { Cuenca del Canal (Tejera } \text { et } \\
\text { al., 1995) }\end{array}$ & 145 & $\begin{array}{l}\text { Muestreo en horas de la } \\
\text { mañana (no especifica). }\end{array}$ \\
\hline $\begin{array}{l}\text { Parque Nacional Chagres } \\
\text { (Fundación Chagres, 2020) }\end{array}$ & 396 & -- \\
\hline $\begin{array}{l}\text { Parque Nacional Soberanía } \\
\text { (Gaceta Oficial, 2017) }\end{array}$ & 402 & -- \\
\hline $\begin{array}{l}\text { Comunidad de Quebrada } \\
\text { Ancha (Contreras y } \\
\text { Yanguez, 2017) }\end{array}$ & 25 & $\begin{array}{l}\text { Entrevista a un grupo de } \\
\text { personas. }\end{array}$ \\
\hline
\end{tabular}


Entre los diferentes taxa el orden Passeriformes fue el mejor representado con 66 especies y 15 familias (Cuadro 2). En términos generales se espera que el orden Passeriformes sea el orden mejor representado durante cualquier estudio de aves de tierra firme en Panamá, ya que este orden incluye la mayor cantidad de especies dentro del país (Araúz y Gonzáles, 2010).

Cuadro 2. Riqueza de familias y especies por orden.

\begin{tabular}{|l|c|c|}
\hline Ordenes & Número de familias & Número de especies \\
\hline Tinamiformes & 1 & 2 \\
\hline Galliformes & 2 & 4 \\
\hline Columbiformes & 1 & 4 \\
\hline Cuculiformes & 1 & 1 \\
\hline Caprimulgiformes & 3 & 6 \\
\hline Charadriiformes & 1 & 1 \\
\hline Pelecaniformes & 1 & 2 \\
\hline Cathartiformes & 1 & 2 \\
\hline Accipitriformes & 2 & 9 \\
\hline Strigiformes & 1 & 2 \\
\hline Trogoniformes & 1 & 1 \\
\hline Coraciiformes & 1 & 2 \\
\hline Galbuliformes & 2 & 4 \\
\hline Piciformes & 4 & 8 \\
\hline Falconiformes & 1 & 2 \\
\hline Psittaciformes & 1 & 3 \\
\hline Passeriformes & 15 & 66 \\
\hline Totales & & \\
\hline Ordenes: $\mathbf{1 8}$ & Familias: 37 & Especies: 119 \\
\hline
\end{tabular}

Especies residentes

Se registraron 90 especies residentes divididas en 35 familias y 17 órdenes (Cuadro 1, Anexo 1). Estas aves representan el $76 \%$ de las especies registradas. El orden Passeriformes fue el más representativo con 44 especies, seguido de Piciformes con 8 especies y los órdenes, Galliformes, Columbiformes y Apodiformes con 4 especies cada uno. El resto de los órdenes registro menos de tres especies residentes.

Guevara, N. 
Entre las familias Thraupidae y Tyrannidae se registró la mayor diversidad con 13 y 7 especies, respectivamente. Entre las especies más comunes registradas estuvieron la tangara dorsirroja (Ramphocelus dimidiatus), la tangara azuleja (Thraupis episcopus) y el bienteveo grande (Pitangus sulphuratus), también se registraron especies comunes de la familia Psittacidae como el perico barbinaranja (Brotogeirs jugularis). Según Angehr \& Dean (2010) y Ridgely y Gwynne (1993) todas las especies residentes registradas son categorizadas como especies comunes y poseen dentro de su posible rango de distribución la provincia de Colón y el distrito de Santa Rita.

Especies Migratorias

Se registraron 29 especies migratorias divididas en nueve familias y tres órdenes. Estas aves representan el 24\% de las especies registradas (Cuadro 3). El orden Passeriformes fue el más representativo con 22 especies, seguido de Accipitriformes con seis especies.

Entre las familias Tyrannidae y Parulidae se registró la mayor diversidad con seis especies cada una. Entre las especies más comunes registradas estuvieron la tangara veranera (Piranga rubra), el mosquerito verdoso (Empidonax virescens) y el gavilán aludo (Buteo platypterus).

Todas las especies migratorias fueron registradas durante los meses de octubre del 2019 a febrero del 2020, época migratoria correspondientes a la migración invernal boreal dada aproximadamente entre los meses de noviembre a abril (Howell \& Webb, 1995). Durante esta época las especies migratorias viajan a las zonas tropicales en busca de alimento el cual escaseará en la región neártica debido al extremo frío provocado por el invierno, por un periodo de seis meses estas especies formaran parte de las comunidades tropicales de aves (Ocampo, 2010). Durante estos meses las especies migratorias pueden habitar diferentes zonas en el Istmo de Panamá (Angehr \& Dean, 2010).

Cabe señalar que durante el presente estudio se registró un individuo de Reinita cerúlea (Setophaga cerulea), la cual es una especie en peligro de extinción debido a la degradación del hábitat por el cambio de uso de la tierra (Birdlife, 2019) lo que provoca la perdida de especies arbóreas claves a causas de enfermedades y al aumento del parasitismo en áreas 
más pequeñas del bosque, lo que provoca una disminución de sus individuos debido a la poca efectividad en la nidificación (Hamel, 2000). Además de la tala completa de bosques para la siembra y producción de especies plantas consumibles (Santander et al., 2012), en el caso de Panamá, como el arroz y el frijol.

La presencia de aves migratorias en Sierra Llorona podría deberse a que en general la mayoría de las aves migratorias del norte evitan el interior del bosque y prefieren los bordes, rastrojos y áreas afectadas por el hombre, como claros y jardines (Ridgely y Gwynne, 1993). Además, muchas de estas especies realizan vuelos migratorios altitudinales siguiendo cadenas montañosas (Serrano y Garitano, 1996) entro los 500 a 2,000 metros (Deinlein, 1999). Durante este viaje las especies buscan lugares próximos en busca de refugio, descanso y alimento, evitando de no ser necesario descender a tierras bajas, lo que provoca un gasto energético mayor (Arosemena, 2006). Debido a que en gran medida las áreas de La Sierra Llorona están afectadas por la actividad humana, y esta posee una altura aproximada a los 300 metros podemos encontrar una cantidad considerable de especies migratorias, las cuales probablemente visitan esta zona próxima a alguna ruta de migración encontrado espacios propicios para descansar y alimentarse.

Cuadro 3. Especies migratorias, Sierra Llorona, provincia de Colón.

\begin{tabular}{|c|c|c|}
\hline Órdenes y familias & Especies & Nombre común \\
\hline \multicolumn{3}{|l|}{ Orden Accipitriformes } \\
\hline Familia Pandionidae & Pandion haliaetus & Águila pescadora \\
\hline \multirow[t]{5}{*}{ Familia Accipitridae } & Elanoides forficatus & Elanio tijereta \\
\hline & Ictinia mississippiensis & Elanio migratorio \\
\hline & Ictinia plumbea & Elanio plomizo \\
\hline & Buteo platypterus & Gavilán aludo \\
\hline & Buteo swainsoni & Gavilán de Swainson \\
\hline \multicolumn{3}{|l|}{ Orden Falconiformes } \\
\hline Familia Falconidae & Falco peregrinus & Halcón peregrino \\
\hline \multicolumn{3}{|l|}{ Orden Passeriformes } \\
\hline \multirow[t]{3}{*}{ Familia Tyrannidae } & Myiarchus crinitus & Copetón viajero \\
\hline & $\begin{array}{l}\text { Myiodynastes } \\
\text { luteiventris }\end{array}$ & $\begin{array}{l}\text { Mosquero } \\
\text { ventiazufrado }\end{array}$ \\
\hline & Tyrannus tyrannus & Tirano norteño \\
\hline
\end{tabular}




\begin{tabular}{|c|c|c|}
\hline & Contopus virens & Pibi oriental \\
\hline & Empidonax virescens & Mosquerito verdoso \\
\hline & Empidonax traillii & Mosquerito de Sauces \\
\hline \multirow[t]{3}{*}{ Familia Vireonidae } & Vireo flavoviridis & Vireo verdiamarillo \\
\hline & Vireo olivaceus & Vireo ojirrojo \\
\hline & Vireo philadelphicus & Vireo de Philadelphia \\
\hline \multirow[t]{2}{*}{ Familia Hirundinidae } & Riparia riparia & Golondrina ribereña \\
\hline & $\begin{array}{l}\text { Petrochelidon } \\
\text { pyrrhonota }\end{array}$ & Golondrina risquera \\
\hline Familia Icteridae & Icterus galbula & Bolsero de Baltimore \\
\hline \multirow[t]{6}{*}{ Familia Parulidae } & Oreothlypis peregrina & Reinita verdilla \\
\hline & Setophaga ruticilla & Candelita norteña \\
\hline & Setophaga cerulea & Reinita cerúlea \\
\hline & Setophaga castanea & Reinita pechicastañea \\
\hline & Setophaga petechia & Reinita amarilla \\
\hline & $\begin{array}{l}\text { Setophaga } \\
\text { pensylvanica }\end{array}$ & Reinita flanquicastañea \\
\hline \multirow[t]{4}{*}{ Familia Cardinalidae } & Piranga rubra & Tangara veranera \\
\hline & Piranga olivacea & Tangara escarlata \\
\hline & $\begin{array}{l}\text { Pheucticus } \\
\text { ludovicianus }\end{array}$ & $\begin{array}{l}\text { Picogrueso } \\
\text { pechirrosado }\end{array}$ \\
\hline & Passerina caerula & Azulillo Indigo \\
\hline \multicolumn{3}{|l|}{ Totales: } \\
\hline Ordenes: 3 & Familias: 9 & $\begin{array}{l}\text { Géneros: 19, Especies: } \\
29\end{array}$ \\
\hline
\end{tabular}

Especies importante para la conservación

Se registraron 24 especies de importancia para la conservación, lo que representa el 20\% de las aves registradas (Cuadro 4). Entre las especies observadas, 24 están protegidas por las leyes panameñas de vida silvestre; siendo 22 especies consideras vulnerables (VU) y dos especies en peligro (EN). Según la IUCN cinco especies están categorizadas como de importancia para la conservación; siendo una especie categorizada como vulnerable (VU) y cuatro bajo la categoría de casi amenazada (NT). 
Entre las especies registradas podemos destacar al Pavón grande (Crax rubra) la cual esta categorizada como En peligro (EN) por las leyes panameñas de vida silvestre y Vulnerable (VU) por la IUCN, esto debido a que es una especie poco común de observar, restringida a áreas protegidas y muy remotas (eBird, 2020). Las principales amenazas que enfrenta esta especie son la cacería, fragmentación y destrucción de su hábitat (Arango, 2016).

Cuadro 4. Aves importantes en conservación, Sierra Llorona, provincia de Colón.

\begin{tabular}{|c|c|c|c|c|}
\hline TAXA & Especies & $\begin{array}{l}\text { Nombre } \\
\text { común }\end{array}$ & MA & IUCN \\
\hline \multicolumn{5}{|l|}{ Orden Tinamiformes } \\
\hline Familia Tinamidae & Tinamus major & $\begin{array}{l}\text { Tinamú } \\
\text { grande }\end{array}$ & VU & NT \\
\hline \multicolumn{5}{|l|}{ Orden: Galliformes } \\
\hline \multirow[t]{2}{*}{ Familia Cracidae } & $\begin{array}{l}\text { Penelope } \\
\text { purpurascens }\end{array}$ & Pava crestada & VU & $\mathrm{LC}$ \\
\hline & Crax rubra & Pavón grande & EN & VU \\
\hline $\begin{array}{c}\text { Familia } \\
\text { Odontophoridae }\end{array}$ & $\begin{array}{l}\text { Odontophorus } \\
\text { gujanensis }\end{array}$ & $\begin{array}{l}\text { Codorniz } \\
\text { jaspeada }\end{array}$ & VU & NT \\
\hline \multicolumn{5}{|c|}{ Orden Caprimulgiformes } \\
\hline \multirow[t]{4}{*}{ Familia Trochilidae } & $\begin{array}{l}\text { Florisuga } \\
\text { mellivora }\end{array}$ & $\begin{array}{l}\text { Jacobino } \\
\text { nuquiblanco }\end{array}$ & VU & $\mathrm{LC}$ \\
\hline & Glaucis hirsutus & $\begin{array}{l}\text { Ermitaño } \\
\text { pechicanelo }\end{array}$ & VU & $\mathrm{LC}$ \\
\hline & $\begin{array}{l}\text { Phaethornis } \\
\text { longirostris }\end{array}$ & $\begin{array}{l}\text { Ermitaño } \\
\text { piquilargo }\end{array}$ & VU & $\mathrm{NE}$ \\
\hline & Amazilia tzacatl & $\begin{array}{l}\text { Amazilia } \\
\text { colirrufa }\end{array}$ & VU & $\mathrm{NE}$ \\
\hline \multicolumn{5}{|l|}{ Orden Accipitriformes } \\
\hline Familia Pandionidae & Pandion haliaetus & $\begin{array}{l}\text { Águila } \\
\text { pescadora }\end{array}$ & VU & $\mathrm{LC}$ \\
\hline \multirow[t]{2}{*}{ Familia Accipitridae } & $\begin{array}{l}\text { Elanoides } \\
\text { forficatus }\end{array}$ & Elanio tijereta & VU & $\mathrm{LC}$ \\
\hline & $\begin{array}{l}\text { Ictinia } \\
\text { mississippiensis }\end{array}$ & $\begin{array}{l}\text { Elanio } \\
\text { migratorio }\end{array}$ & VU & $\mathrm{LC}$ \\
\hline
\end{tabular}




\begin{tabular}{|c|c|c|c|c|}
\hline & Ictinia plumbea & $\begin{array}{l}\text { Elanio } \\
\text { plomizo }\end{array}$ & VU & $\mathrm{LC}$ \\
\hline & $\begin{array}{l}\text { Geranospiza } \\
\text { caerulescens }\end{array}$ & $\begin{array}{l}\text { Gavilán } \\
\text { zancón }\end{array}$ & VU & LC \\
\hline & Buteo platypterus & Gavilán aludo & VU & LC \\
\hline & Buteo swainsoni & $\begin{array}{l}\text { Gavilán de } \\
\text { Swainson }\end{array}$ & VU & LC \\
\hline \multicolumn{5}{|l|}{ Orden Strigiformes } \\
\hline \multirow[t]{2}{*}{ Familia Strigidae } & $\begin{array}{l}\text { Pulsatrix } \\
\text { perspicillata }\end{array}$ & $\begin{array}{l}\text { Búho de } \\
\text { anteojos }\end{array}$ & VU & LC \\
\hline & $\begin{array}{l}\text { Megascops } \\
\text { choliba }\end{array}$ & $\begin{array}{l}\text { Autillo } \\
\text { tropical }\end{array}$ & VU & LC \\
\hline \multicolumn{5}{|l|}{ Orden: Falconiformes } \\
\hline Familia Falconidae & Falco peregrinus & $\begin{array}{l}\text { Halcón } \\
\text { peregrino }\end{array}$ & VU & LC \\
\hline \multicolumn{5}{|l|}{ Orden Psittaciformes } \\
\hline \multirow[t]{3}{*}{ Familia Psittacidae } & $\begin{array}{l}\text { Brotogeris } \\
\text { jugularis }\end{array}$ & $\begin{array}{l}\text { Perico } \\
\text { barbinaranja }\end{array}$ & VU & LC \\
\hline & $\begin{array}{l}\text { Amazona } \\
\text { autumnalis }\end{array}$ & $\begin{array}{l}\text { Loro } \\
\text { frentirrojo }\end{array}$ & VU & LC \\
\hline & $\begin{array}{l}\text { Amazona } \\
\text { ochrocephala }\end{array}$ & $\begin{array}{c}\text { Loro } \\
\text { coroniamarillo }\end{array}$ & EN & LC \\
\hline \multicolumn{5}{|l|}{ Orden Piciformes } \\
\hline \multirow[t]{2}{*}{ Familia Ramphastidae } & $\begin{array}{l}\text { Ramphastos } \\
\text { sulfuratus }\end{array}$ & & VU & LC \\
\hline & $\begin{array}{l}\text { Ramphastos } \\
\text { ambiguus }\end{array}$ & & VU & NT \\
\hline \multicolumn{5}{|l|}{ Orden Passeriformes } \\
\hline Familia Parulidae & $\begin{array}{l}\text { Setophaga } \\
\text { cerulea }\end{array}$ & $\begin{array}{l}\text { Reinita } \\
\text { cerúlea }\end{array}$ & VU & NT \\
\hline \multicolumn{5}{|l|}{ Totales: } \\
\hline Ordenes: 9 & Familias: 11 & $\begin{array}{l}\text { Géneros: } 19 \\
\text { Especies: } 24\end{array}$ & & \\
\hline
\end{tabular}

Leyenda: MA: Ministerio de Ambiente de Panamá (VU: vulnerable, EN: en peligro), IUCN: Unión Internacional para la Conservación de la Naturaleza (VU: vulnerable, NT: casi amenazado, NE: no evaluada, LC: Preocupación menor). Nombres científicos, nombres comunes y arreglo taxonómico según AUDUBON (2019). 


\section{CONCLUSIÓN}

A pesar de su ubicación e intervención humana, Sierra Llorona sirve de hábitat a una considerable variedad de aves, albergando el 12\% de las aves conocidas para Panamá.

Además, sirve de hábitat a una considerable cantidad de especies migratorias, lo que en cierto grado demuestra su grado de perturbación, ya que muchas especies de este grupo prefieren áreas abiertas y perturbadas por la actividad humana, evitando bordes de bosques durante su paso migratorio en el Neotrópico.

Este sitio también sirve de hogar a una gran variedad de especies bajo algún grado de amenaza, especialmente de aves pocos comunes como el pavón grande y la reinita cerúlea, las cuales están gravemente afectadas por la pérdida de hábitat.

La presencia de una gran variedad de aves en Sierra Llorona es indicativa de la recuperación de las zonas boscosas y hábitat del lugar, lo cual también puede ser provechoso para otros grupos de animales diferentes a las aves.

\section{AGRADECIMIENTOS}

A la Sociedad Mastozoológica de Panamá (SOMASPA) por hacerme parte del proyecto del Corredor Biológico del Jaguar, lo que me permitió visitar diferentes áreas de Sierra Llorona, logrando realizar los muestreos y obtención de datos de una manera eficaz, visitando un área representativa del lugar.

Al Doctor Rafael Samudio Jr. y a la Magister Julieta E. Carrión de Samudio por el apoyo en la revisión y corrección del presente documento para su mejor presentación.

A los propietarios, administradores y encargados de los terrenos abarcados en el sitio de estudio, los cuales consistieron en terrenos y reservas privadas. Lo que me permitió realizar muestreos en zonas boscosas. 


\section{REFERENCIAS}

ANAM (Autoridad Nacional del Ambiente). 2010. Atlas Ambiental de la República de Panamá. Primera Versión. Gobierno Nacional de la República de Panamá. 190 pp.

Angehr, G., \& Dean, R. 2010. The Birds of Panama A Field Guide. Zona Tropical Editor in Chief: John K. McCuen. Book design: Zona Creativa S.A., Publication from Comstock Publishing Associates. división of Cornell University Press. Ithaca. 456 pp.

Arango, C. 2016. Pavón Chocoano (Crax rubra). Wiki Aves de Colombia. (C. Arango, Editor). Universidad Icesi. Cali, Colombia. Recuperado el 18 de agosto de 2020, de https://www.icesi.edu.co/wiki_aves_colombia/tikiindex.php?page_ref_id=1832

Araúz, J. y González, D. 2010. Aves de Cerro Canajagua, Provincia de Los Santos, Panamá. Universidad de Panamá. Tecnociencia 2010, Vol. 12, No. 2. 138 pp.

Arosemena, M. 2006. Atlas Visual de la Ciencia. Aves. Editorial Sol 90. $95 \mathrm{pp}$.

AUDUBON. 2019. Lista de aves de Panamá. Comité de registros, Sociedad Audubon de Panamá. 16 pp.

BirdLife International. 2018. El Estado de conservación de las aves del mundo: tomando el pulso de nuestro planeta. Cambridge University, Reino Unido: BirdLife International. 80 pp. Recuperado el 14 de julio de 2020, de http://datazone.birdlife.org/userfiles/docs/SOWB2018_es.pdf.

BirdLife International. 2019. Setophaga cerulea. The IUCN Red List of Threatened Species 2019: e.T22721740A153691320. Recuperado el 10 de octubre de 2020, de https://dx.doi.org/10.2305/IUCN.UK.20193.RLTS.T22721740A153691320. 
Contreras, M. y Yanguez, A. 2017. Caracterización de los usos de vertebrados silvestres en la comunidad de Quebrada Ancha, Colón (Panamá). Centros: Revista Científica Universitaria, 6(2), 18-30. Recuperado el 18 de agosto de 2020, de http://revistas.up.ac.pa/index.php/centros/article/view/4 .

Deinlein, M. 1999. Conceptos básicos sobre las aves migratorias Neotropicales. Smithsonian's National Zoo \& Conservation Biology Institute.

Del Coro, M., Carol, B., Humberto, B., Peter, J.B., Gregory, S.B., Andrew, R.C., Ashley, A.D., Dean, W.D., Wendy, E.E., Mary, G., Eduardo, E., Judith, A.K., Elizabeth. A.K., Arvind, O.P., Terrell, D.R., Vicente, R.C., Kenneth, V.R., Janet, M.R., Eduardo, S.C., Rosa, M.V. y Tom, W. 2019. Conservando a nuestras aves compartidas: La visión trinacional de compañeros en vuelo para la conservación de las aves terrestres. Cornell Lab of Ornithology: Ithaca, NY. 52 pp.

eBird. 2020. Pavón grande (Crax rubra). eBird Basic Dataset. Cornell Lab of Ornithology, Ithaca, New York. Recuperado el 18 de agosto de 2020, de https://ebird.org/species/grecur1?siteLanguage=es_PA.

ETESA (Empresa de Transmisión Eléctrica). 2007. Hidrometeorología Panamá. Mapa de Clasificación Climática (según KÖPPEN). Recuperado el 19 de mayo de 2020, de http://www.hidromet.com.pa/Mapas/Mapa_Clasificacion_Climatica_K OPPEN_2007_Panama.pdf

Fundación Chagres. 2020. Fundación Parque Nacional Chagres, Área de incidencia. Recuperado el 22 de mayo de 2020, de http://www.fundacionchagres.org/\#: :text=El\%20parque\%20nacional $\% 20$ Chagres\%2C\%20tiene,y\%2079\%20especies\%20de\%20anfibios.

Gaceta Oficial Digital No. 28187-A del 29 de diciembre de 2016. Ministerio de Ambiente (Miambiente). República de Panamá. 50 pp.

Gaceta Oficial Digital No. 28254-B del 7 de abril de 2017. Ministerio de Ambiente (Miambiente). República de Panamá. 379 pp. 
Gaceta Oficial Digital No. 28285-A del 24 de mayo de 2017. Ministerio de Ambiente (Miambiente). Republica de Panamá. 192pp.

Hamel, P. B. 2000. Cerulean Warbler (Dendroica cerulea). In The Birds of North America, No. 511 (A. Poole and F. Gill, eds.). The Birds of North America, Inc., Philadelphia, PA. 20 pp.

Heckadon, S.M., Ibañez, R. y Condit, R. 1999. La Cuenca del Canal: deforestación, urbanización y contaminación. Proyecto de monitoreo de la Cuenca del Canal de Panamá, Sumario ejecutivo del informe final. Autoridad del Medio Ambiente (ANAM). 125 pp.

Holdridge, L. 1979. Ecología basada en zonas de vida. Traducción de la primera edición. Instituto Interamericano de Ciencias Agrícolas, San José, Costa Rica. 216 pp.

Howell, S.N.G., \& Webb, S. 1995. A Guide to the Birds of México and Northern Central America. Oxford Universidad Press, Oxford, 851 pp.

The IUCN Red List of Threatened Species. Recuperado el 15 de agosto de 2020, de https://www.iucnredlist.org/.

Lepage, D. 2020. Checklist of the birds of Panama (Central). Avibase, the world bird database. Recuperado el 9 de octubre del 2020, de https://avibase.bsceoc.org/checklist.jsp?lang=EN\&region $=$ pace \&list $=\mathrm{c}$ lements\&format $=1$.

Moreno, C. E. 2001. Métodos para medir la biodiversidad. M\&TManuales y Tesis SEA, vol.1. Zaragoza, 84 pp.

Ocampo, P.N. 2010. El fenómeno de la migración en aves: una mirada desde la Orinoquia. Orinoquia, 14(2), 188-200. 13 pp. Recuperado el 10 de octubre de 2020, de http://www.scielo.org.co/scielo.php?script=sci_arttext\&pid=S012137092010000200009\&lng=en\&tlng=es.

Pereyra, J.A. 2019. Importancia de nuestras aves. Revista de Ornitología Neotropical. Hornero 006 (02): 254-261 pp. 
Polanco, J. M., Ospina, A.D., Arango, D.G., Snaider, J.G. y Humberto, M.G. 2015. Efectividad de las redes de niebla para determinarla riqueza de aves en un bosque montano de los Andes Centrales (Salento, Quindío, Colombia). Revista de Investigaciones. Universidad de Quindío. Colombia, 27(1): 75-88; 2015.

Ralph, J.C., Greuoel G.R., Pyle, P., Martín, T.E., Desante, D.F. y Milá, B. 1996. Manual de métodos de campo para el monitoreo de aves terrestres. United States Depart. of Agricul., General Technical Report. $45 \mathrm{pp}$.

Ridgely, R. y Gwynne, J. 1993. Guía de las aves de Panamá, incluyendo Costa Rica, Nicaragua y Honduras. Primera edición en español. Asociación Nacional para la Conservación de la Naturaleza (ANCON). 614 pp.

Santander, T., Adrián, G. y Estaban, G. 2012. Conservando el hábitat invernal de la reinita cerúlea (Setophaga cerúlea) en Ecuador. Ornitología Neotropical, 23: 343-350, 2012.9 pp.

Serrano, V. y Garitano, A. 1996. Biodiversidad de Aves a nivel del paisaje en bosques Andinos y estudios de sus procesos dinámicos. Fundación Cali para la Promoción de la Investigación y la Tecnología. $10 \mathrm{pp}$.

Stiles, F.G. y Rosselli, L. 1998. Inventario de las Aves de un Bosque Altoandino: Comparación de dos Métodos. Caldasia 20 (1): 29-43.

SOMASPA (Sociedad Mastozoológica de Panamá). 2019. Felinos del Corredor Biológico Colón del jaguar: silenciosos caminantes del bosque. Serie de Folletos de Biodiversidad, SOMASPA 2019.

Tejera, V., Ibáñez, R. y Arosemena, G. 1995. El Inventario Biológico del Canal de Panamá II. El Estudio Ornitológico, Herpetológico y Mastozoológico. Scientia: 5 -281.

Villareal H., Álvarez, M., Córdoba, S., Escobar, F., Fagua, G., Gast, F., Mendoza, H., Ospina, M. y Umaña, A.M. 2004. Manual de métodos para el desarrollo de inventarios de biodiversidad. Programa de 
inventarios de Biodiversidad. Instituto de Investigación de Recursos Biológicos Alexander von Humboldt. Bogotá, Colombia. 236 pp.

Anexo 1. Especies de aves registradas en Sierra Llorona, provincia de Colón.

\begin{tabular}{|c|c|c|}
\hline $\begin{array}{l}\text { ORDENES Y } \\
\text { FAMILIAS }\end{array}$ & ESPECIES & NOMBRE COMÚN \\
\hline \multicolumn{3}{|l|}{ Orden Tinamiformes } \\
\hline \multirow{2}{*}{ Familia Tinamidae } & Tinamus major & Tinamú Grande \\
\hline & Crypturellus soui & Tinamú Chico \\
\hline \multicolumn{3}{|l|}{ Orden Galliformes } \\
\hline \multirow[t]{3}{*}{ Familia Cracidae } & Ortalis cinereiceps & $\begin{array}{l}\text { Chachalaca } \\
\text { Cabecigrís }\end{array}$ \\
\hline & $\begin{array}{l}\text { Penelope } \\
\text { purpurascens }\end{array}$ & Pava Crestada \\
\hline & Crax rubra & Pavón grande \\
\hline $\begin{array}{l}\text { Familia } \\
\text { Odontophoridae }\end{array}$ & $\begin{array}{l}\text { Odontophorus } \\
\text { gujanensis }\end{array}$ & Codorniz Jaspeada \\
\hline \multicolumn{3}{|l|}{ Orden Columbiformes } \\
\hline \multirow[t]{4}{*}{ Familia Columbidae } & Columba livia & Paloma doméstica \\
\hline & $\begin{array}{l}\text { Patagioenas } \\
\text { cayennensis }\end{array}$ & Paloma Colorada \\
\hline & Columbina talpacoti & Tortolita Rojiza \\
\hline & Leptotila verreauxi & Paloma Rabiblanca \\
\hline \multicolumn{3}{|l|}{ Orden Cuculiformes } \\
\hline Familia Cuculidae & Crotophaga ani & Garrapatero Piquiliso \\
\hline \multicolumn{3}{|l|}{$\begin{array}{l}\text { Orden } \\
\text { Caprimulgiformes }\end{array}$} \\
\hline $\begin{array}{l}\text { Familia } \\
\text { Caprimulgidae }\end{array}$ & Nyctidromus albicollis & Tapacamino Común \\
\hline Familia Nyctibiidae & Nyctibius griseus & Nictibio Común \\
\hline \multirow[t]{4}{*}{ Familia Trochilidae } & Florisuga mellivora & Jacobino Nuquiblanco \\
\hline & Glaucis hirsutus & Ermitaño Pechicanelo \\
\hline & $\begin{array}{l}\text { Phaethornis } \\
\text { longirostris }\end{array}$ & Ermitaño Piquilargo \\
\hline & Amazilia tzacatl & Amazilia Colirrufa \\
\hline
\end{tabular}




\begin{tabular}{|c|c|c|}
\hline $\begin{array}{l}\text { Orden } \\
\text { Charadriiformes }\end{array}$ & & \\
\hline Familia Charadriidae & Vanellus chilensis & Tero Sureño \\
\hline Orden Pelecaniformes & & \\
\hline Familia Ardeidae & Tigrisoma lineatum & Garza-Tigre Castaña \\
\hline & Ardea alba & Garceta Grande \\
\hline Orden Cathartiformes & & \\
\hline Familia Cathartidae & Coragyps atratus & Gallinazo Negro \\
\hline & Cathartes aura & Gallinazo Cabecirrojo \\
\hline Orden Accipitriformes & & \\
\hline Familia Pandionidae & Pandion haliaetus & Águila pescadora \\
\hline Familia Accipitridae & Elanoides forficatus & Elanio tijereta \\
\hline & $\begin{array}{l}\text { Ictinia } \\
\text { mississippiensis }\end{array}$ & Elanio migratorio \\
\hline & Ictinia plumbea & Elanio plomizo \\
\hline & $\begin{array}{l}\text { Geranospiza } \\
\text { caerulescens }\end{array}$ & Gavilán Zancón \\
\hline & Rupornis magnirostris & Gavilán Caminero \\
\hline & Pseudastur albicollis & Gavilán Blanco \\
\hline & Buteo platypterus & Gavilán Aludo \\
\hline & Buteo swainsoni & Gavilán de Swainson \\
\hline Orden Strigiformes & & \\
\hline Familia Strigidae & Pulsatrix perspicillata & Búho de Anteojos \\
\hline & Megascops choliba & Autillo tropical \\
\hline Orden Trogoniformes & & \\
\hline Familia Trogonidae & Trogon massena & Trogón Colipizarra \\
\hline Orden Coraciiformes & & \\
\hline Familia Momotidae & Momotus subrufescens & Momoto Gritón \\
\hline & $\begin{array}{l}\text { Electron } \\
\text { platyrhynchum }\end{array}$ & Momoto Piquiancho \\
\hline Orden Galbuliformes & & \\
\hline Familia Bucconidae & $\begin{array}{l}\text { Notharchus } \\
\text { hyperrhynchus }\end{array}$ & Buco Cuelliblanco \\
\hline & Notharchus tectus & Buco Pinto \\
\hline & $\begin{array}{l}\text { Malacoptila } \\
\text { panamensis }\end{array}$ & Buco Bigotiblanco \\
\hline
\end{tabular}




\begin{tabular}{|c|c|c|}
\hline Familia Galbulidae & Jacamerops aureus & Jacamar Grande \\
\hline \multicolumn{3}{|l|}{ Orden Piciformes } \\
\hline Familia Alcedinidae & Chloroceryle amazona & $\begin{array}{l}\text { Martín Pescador } \\
\text { Amazónico }\end{array}$ \\
\hline \multirow[t]{3}{*}{ Familia Ramphastidae } & $\begin{array}{l}\text { Pteroglossus } \\
\text { torquatus }\end{array}$ & Tucancillo Collarejo \\
\hline & Ramphastos sulfuratus & Tucán Pico Iris \\
\hline & Ramphastos ambiguus & Tucán Gorguiamarillo \\
\hline \multirow[t]{4}{*}{ Familia Picidae } & Melanerpes pucherani & Carpintero Carinegro \\
\hline & $\begin{array}{l}\text { Melanerpes } \\
\text { rubricapillus }\end{array}$ & $\begin{array}{l}\text { Carpintero } \\
\text { Coronirrojo }\end{array}$ \\
\hline & Dryocopus lineatus & Carpintero Lineado \\
\hline & $\begin{array}{l}\text { Campephilus } \\
\text { haematogaster }\end{array}$ & Carpintero carminoso \\
\hline \multicolumn{3}{|l|}{ Orden Falconiformes } \\
\hline \multirow[t]{2}{*}{ Familia Falconidae } & Milvago chimachima & $\begin{array}{l}\text { Caracara } \\
\text { Cabeciamarilla }\end{array}$ \\
\hline & Falco peregrinus & Halcón Peregrino \\
\hline \multicolumn{3}{|l|}{ Orden Psittaciformes } \\
\hline \multirow[t]{3}{*}{ Familia Psittacidae } & Brotogeris jugularis & Perico Barbinaranja \\
\hline & Amazona autumnalis & Loro Frentirrojo \\
\hline & $\begin{array}{l}\text { Amazona } \\
\text { ochrocephala }\end{array}$ & Loro Coroniamarillo \\
\hline \multicolumn{3}{|l|}{ Orden Passeriformes } \\
\hline \multirow{6}{*}{$\begin{array}{l}\text { Familia } \\
\text { Thamnophilidae }\end{array}$} & Cymbilaimus lineatus & Batará Lineado \\
\hline & $\begin{array}{l}\text { Thamnophilus } \\
\text { atrinucha }\end{array}$ & Batará Coroninegro \\
\hline & $\begin{array}{l}\text { Epinecrophylla } \\
\text { fulviventris }\end{array}$ & $\begin{array}{l}\text { Hormiguerito } \\
\text { Leonado }\end{array}$ \\
\hline & $\begin{array}{l}\text { Microrhopias } \\
\text { quixensis }\end{array}$ & $\begin{array}{l}\text { Hormiguerito } \\
\text { Alipunteado }\end{array}$ \\
\hline & Poliocrania exsul & $\begin{array}{l}\text { Hormiguero } \\
\text { Dorsicastaño }\end{array}$ \\
\hline & Hylophylax naevioides & Hormiguero Collarejo \\
\hline Familia Furnariidae & $\begin{array}{l}\text { Xiphorhynchus } \\
\text { susurrans }\end{array}$ & $\begin{array}{l}\text { Trepatroncos } \\
\text { Chocolate }\end{array}$ \\
\hline
\end{tabular}

Tecnociencia, Vol. 23, $N^{\circ} 1$ 


\begin{tabular}{|c|c|c|}
\hline Familia Pipridae & Manacus vitellinus & Saltarín Cuellidorado \\
\hline Familia Cotingidae & Querula purpurata & $\begin{array}{l}\text { Quérula } \\
\text { Gorguimorada }\end{array}$ \\
\hline \multirow[t]{13}{*}{ Familia Tyrannidae } & Todirostrum cinereum & Espatulilla Común \\
\hline & Elaenia flavogaster & Elenia Penachuda \\
\hline & $\begin{array}{l}\text { Myiarchus } \\
\text { panamensis }\end{array}$ & Copetón Panameño \\
\hline & Myiarchus crinitus & Copetón Viajero \\
\hline & Pitangus sulphuratus & Bienteveo Grande \\
\hline & $\begin{array}{l}\text { Myiozetetes } \\
\text { cayanensis }\end{array}$ & Mosquero Alicastaño \\
\hline & $\begin{array}{l}\text { Myiodynastes } \\
\text { maculatus }\end{array}$ & Mosquero Rayado \\
\hline & $\begin{array}{l}\text { Myiodynastes } \\
\text { luteiventris }\end{array}$ & $\begin{array}{l}\text { Mosquero } \\
\text { Ventriazufrado }\end{array}$ \\
\hline & $\begin{array}{l}\text { Tyrannus } \\
\text { melancholicus }\end{array}$ & Tirano Tropical \\
\hline & Tyrannus tyrannus & Tirano Norteño \\
\hline & Contopus virens & Pibí Oriental \\
\hline & Empidonax virescens & Mosquerito Verdoso \\
\hline & Empidonax traillii & Mosquerito de Sauces \\
\hline \multirow[t]{3}{*}{ Familia Vireonidae } & Vireo philadelphicus & Vireo de Philadelphia \\
\hline & Vireo olivaceus & Vireo Ojirrojo \\
\hline & Vireo flavoviridis & Vireo Verdiamarillo \\
\hline \multirow[t]{3}{*}{ Familia Hirundinidae } & $\begin{array}{l}\text { Stelgidopteryx } \\
\text { ruficollis }\end{array}$ & $\begin{array}{l}\text { Golondrina } \\
\text { Alirrasposa Sureña }\end{array}$ \\
\hline & Riparia riparia & Golondrina Ribereña \\
\hline & $\begin{array}{l}\text { Petrochelidon } \\
\text { pyrrhonota }\end{array}$ & Golondrina Risquera \\
\hline Familia Polioptilidae & $\begin{array}{l}\text { Ramphocaenus } \\
\text { melanurus }\end{array}$ & Soterillo Piquilargo \\
\hline \multirow[t]{4}{*}{ Familia Troglodytidae } & Troglodytes aedon & Sotorrey Común \\
\hline & $\begin{array}{l}\text { Pheugopedius } \\
\text { fasciatoventris }\end{array}$ & Sotorrey Ventrinegro \\
\hline & $\begin{array}{l}\text { Cantorchilus } \\
\text { leucopogon }\end{array}$ & $\begin{array}{l}\text { Sotorrey } \\
\text { Gorguirrayado }\end{array}$ \\
\hline & $\begin{array}{l}\text { Cantorchilus } \\
\text { nigricapillus }\end{array}$ & Sotorrey Castaño \\
\hline
\end{tabular}




\begin{tabular}{|c|c|c|}
\hline Familia Turdidae & Turdus grayi & Mirlo pardo \\
\hline \multirow[t]{3}{*}{ Familia Fringillidae } & Euphonia laniirostris & Eufonia Piquigruesa \\
\hline & Euphonia minuta & Eufonia Ventriblanca \\
\hline & Spinus psaltria & Jilguero Menor \\
\hline \multirow[t]{5}{*}{ Familia Icteridae } & Psarocolius wagleri & $\begin{array}{l}\text { Oropéndola } \\
\text { Cabecicastaña }\end{array}$ \\
\hline & Cacicus uropygialis & $\begin{array}{l}\text { Cacique } \\
\text { Lomiescarlata }\end{array}$ \\
\hline & Icterus chrysater & Bolsero Dorsiamarillo \\
\hline & Icterus galbula & Bolsero de Baltimore \\
\hline & Quiscalus mexicanus & Tordo Coligrande \\
\hline \multirow[t]{6}{*}{ Familia Parulidae } & Oreothlypis peregrina & Reinita verdilla \\
\hline & Setophaga ruticilla & Candelita norteña \\
\hline & Setophaga cerulea & Reinita cerúlea \\
\hline & Setophaga castanea & Reinita pechicastañea \\
\hline & Setophaga petechia & Reinita amarilla \\
\hline & $\begin{array}{l}\text { Setophaga } \\
\text { pensylvanica }\end{array}$ & $\begin{array}{l}\text { Reinita } \\
\text { flanquicastañea }\end{array}$ \\
\hline \multirow[t]{5}{*}{ Familia Cardinalidae } & Piranga rubra & Tangara veranera \\
\hline & Piranga olivacea & Tangara escarlata \\
\hline & Habia fuscicauda & $\begin{array}{l}\text { Tangara-Hormiguera } \\
\text { Gorguirroja }\end{array}$ \\
\hline & $\begin{array}{l}\text { Pheucticus } \\
\text { ludovicianus }\end{array}$ & $\begin{array}{l}\text { Picogrueso } \\
\text { pechirrosado }\end{array}$ \\
\hline & Passerina caerula & Azulillo Indigo \\
\hline \multirow[t]{7}{*}{ Familia Thraupidae } & Thraupis episcopus & Tangara Azuleja \\
\hline & Thraupis palmarum & Tangara Palmera \\
\hline & Stilpnia larvata & $\begin{array}{l}\text { Tangara } \\
\text { Capuchidorada }\end{array}$ \\
\hline & Tangara inornata & Tangara Cenicienta \\
\hline & $\begin{array}{l}\text { Tachyphonus } \\
\text { luctuosus }\end{array}$ & $\begin{array}{l}\text { Tangara } \\
\text { Hombriblanca }\end{array}$ \\
\hline & $\begin{array}{l}\text { Ramphocelus } \\
\text { flammigerus }\end{array}$ & Tangara Lomiflama \\
\hline & $\begin{array}{l}\text { Ramphocelus } \\
\text { dimidiatus }\end{array}$ & Tangara Dorsirroja \\
\hline
\end{tabular}

Tecnociencia, Vol. 23, $N^{\circ} 1$ 


\begin{tabular}{|l|l|l|}
\hline & Cyanerpes cyaneus & Mielero Patirrojo \\
\hline & Dacnis cayana & Dacnis Azul \\
\hline & Tiaris olivaceus & $\begin{array}{l}\text { Semillerito } \\
\text { Cariamarillo }\end{array}$ \\
\hline & Sporophila corvina & Espiguero Variable \\
& Saltador maximus & $\begin{array}{l}\text { Saltor } \\
\text { Gorguianteado }\end{array}$ \\
\hline & Saltator grossus & Picogrueso Piquirrojo \\
\hline Totales: & Familias: 37 & Especies: 119 \\
\hline Ordenes: $\mathbf{1 8}$ & & \\
\hline
\end{tabular}

Clasificación taxonómica y nombres científicos según Lepage (2020), nombres comunes según AUDUBON (2019).

Recibido, 05 de mayo 2020, y aceptado 06 noviembre 2020. 